\title{
PERIODICITY OF SOMOS SEQUENCES
}

\author{
RAPHAEL M. ROBINSON
}

(Communicated by William W. Adams)

\begin{abstract}
Various sequences related to one introduced by Michael Somos are shown to be periodic mod $m$ for every $m$, although the problem remains open for the original sequence. Some observations are made concerning the prime divisors and the rate of growth of certain sequences.
\end{abstract}

\section{INTRODUCTION}

For $k \geqq 4$, by $\operatorname{Somos}(k)$ we shall mean the sequence defined by

$$
\begin{gathered}
a_{0}=a_{1}=\cdots=a_{k-1}=1, \\
a_{n} a_{n-k}=\sum_{i=1}^{[k / 2]} a_{n-i} a_{n-k+i} \quad \text { for } n \geqq k .
\end{gathered}
$$

Somos first introduced the sequence $\operatorname{Somos}(6)$ and in [2] raised the question whether all the terms are integers. Some history of the problem may be found in Gale [1]. It is now known that the terms of $\operatorname{Somos}(k)$ are all integers for $k=4,5,6,7$, but not for $k=8$.

Notice that the sequence $\operatorname{Somos}(k)$ can also be extended backwards and has a center of symmetry at $n=(k-1) / 2$.

By a generalized Somos sequence, we shall mean a sequence where the initial values $a_{0}, a_{1}, \ldots, a_{k-1}$ are positive integers and

$$
a_{n} a_{n-k}=P\left(a_{n-1}, a_{n-2}, \ldots, a_{n-k+1}\right) \text { for } n \geqq k,
$$

where $P$ is a polynomial with positive integer coefficients.

Such a sequence will be said to satisfy condition $(\mathrm{H})$ if when $a_{0}, a_{1}$, $\ldots, a_{k-1}$ are considered as variables, we may write

$$
a_{n}=p_{n}\left(a_{0}, a_{1}, \ldots, a_{k-1}\right) / q_{n}\left(a_{0}, a_{1}, \ldots, a_{k-1}\right),
$$

where $p_{n}$ is a polynomial with positive integer coefficients, but $q_{n}$ is just a product of powers of the variables.

If a sequence satisfies condition $(\mathrm{H})$ and if the initial values are all equal to 1 , then clearly all terms are integers. This condition was introduced by Dean

Received by the editors January 7, 1991 .

1991 Mathematics Subject Classification. Primary 11B37; Secondary 11B50. 
Hickerson to prove that the terms of $\operatorname{Somos}(6)$ are integers, so I named it for him.

The relation $a_{n}=p_{n} / q_{n}$ required by condition $(\mathrm{H})$ is trivial for $n<2 k$. Hickerson showed that to verify the condition in general, it is sufficient to check it for $n=2 k$ and to check that $p_{k}$ is prime to $p_{k+1}, \ldots, p_{2 k}$. The result for all $n>2 k$ then follows by innduction. On the one hand, we have

$$
a_{n}=p_{n-1}\left(a_{1}, \ldots, a_{k}\right) / q_{n-1}\left(a_{1}, \ldots, a_{k}\right),
$$

and on the other hand,

$$
a_{n}=p_{n-k-1}\left(a_{k+1}, \ldots, a_{2 k}\right) / q_{n-k-1}\left(a_{k+1}, \ldots, a_{2 k}\right) \text {. }
$$

Substituting the values of $a_{k}, a_{k+1}, \ldots, a_{2 k}$, we obtain an expression for $a_{n}$ whose denominator is a product of powers of the variables and of $p_{k}$ in the first case and of $p_{k+1}, \ldots, p_{2 k}$ in the second. Since $p_{k}$ is prime to $p_{k+1}, \ldots, p_{2 k}$, the reduced denominator must be a product of powers of the variables.

Condition $(\mathrm{H})$ is known to hold for $\operatorname{Somos}(k)$ when $k=4,5,6,7$. It was checked for $k=6$ by Hickerson, using a computer program. A similar check was made for $k=7$ by Benjamin Lotto. The cases $k=4$ and $k=5$ can be checked by hand.

\section{TWO THEOREMS ON PERIODICITY}

Since the computation of $\operatorname{Somos}(k)$ for $4 \leqq k \leqq 7$ involves division, it is not clear that the terms $a_{n}$, known to be integers, must form a sequence that is periodic mod $m$ for each modulus $m$, although this seems to be the case. Indeed, it is not even clear that periodicity that has been observed must continue. But the first theorem gives information about this.

Theorem 1. If a generalized Somos sequence satisfies condition $(\mathbf{H})$, if $a_{0}, a_{1}, \ldots$, $a_{k-1}$ are prime to $m$, and if

$$
a_{r+n} \equiv a_{n}(\bmod m) \quad \text { for } n=0,1, \ldots, k-1,
$$

then the sequence $a_{n}(\bmod m)$ has period $r$.

Remark. Under the given hypotheses, the terms of the sequence may not all be integers, but they are $m$-adic integers (that is, have denominators prime to $m$ ), and hence may be considered $\bmod m$.

Proof. We have, for all $n$,

$$
a_{n}=p_{n}\left(a_{0}, a_{1}, \ldots, a_{k-1}\right) / q_{n}\left(a_{0}, a_{1}, \ldots, a_{k-1}\right)
$$

and

$$
a_{r+n}=p_{n}\left(a_{r}, a_{r+1}, \ldots, a_{r+k-1}\right) / q_{n}\left(a_{r}, a_{r+1}, \ldots, a_{r+k-1}\right) .
$$

Here the numerators are congruent $\bmod m$, the denominators are congruent mod $m$, and, by condition $(\mathbf{H})$, the denominators are prime to $m$. Hence $a_{r+n} \equiv a_{n}(\bmod m)$.

This theorem enables us to recognize periodicity mod $m$ for many sequences, including $\operatorname{Somos}(k)$ for $k=4,5,6,7$. But can we predict periodicity? The second theorem provides some information. 
Theorem 2. For $k=4$ or $k=5$, $\operatorname{Somos}(k)$ is periodic $\bmod m$ for every $m$. Proof. We shall make use of the fact that, in these sequences, $a_{i}$ and $a_{j}$ are relatively prime whenever $|i-j| \leqq k$. The proof of this result is postponed to $\S 3$.

Assuming this, it follows that for any prime $p$ the sequence $a_{n}$ must have at least $k$ numbers prime to $p$ between any two multiples of $p$. Hence there are infinitely many blocks of length $k$ of terms prime to $p$. Given any $l$, we can find two of these blocks that are congruent mod $p^{l}$. By Theorem 1, this establishes periodicity mod $p^{l}$ going forward. But we can also read the sequence backwards, so we have pure periodicity. Periodicity mod $m$ follows by factoring $m$ into prime powers.

This method does not work for $k=6$ or $k=7$. I do not know any proof of periodicity for these cases.

\section{Relative primeness in Somos(4) and Somos(5)}

For $k=4$ and $k=5$, it is known, and easily proved by induction, is that two terms $a_{i}$ and $a_{j}$ in $\operatorname{Somos}(k)$ are relatively prime if $|i-j|<k$. Indeed, this was the basis of a simple proof, given by George Bergman, that these sequences have integer terms. But we need the stronger result that $a_{i}$ and $a_{j}$ are relatively prime whenever $|i-j| \leqq k$. that

For $\operatorname{Somos}(4)$, we need to show that $a_{n}$ is prime to $a_{n+4}$. We shall prove

$$
a_{n} a_{n+6}^{2}+a_{n+2}^{2} a_{n+8} \equiv 0 \quad\left(\bmod a_{n+4}\right) .
$$

This shows that if $a_{n}$ is prime to $a_{n+4}$ then $a_{n+4}$ is prime to $a_{n+8}$. Since $a_{n}$ is prime to $a_{n+4}$ for $n=0,1,2,3$, the general result follows by induction.

To simplify the notation, let $b_{i}=a_{n+i}$. Since $b_{i}$ satisfies the same recursion relation as $a_{i}$, we see that

$$
\begin{aligned}
b_{1} b_{5} \cdot b_{3} b_{7} & =\left(b_{2} b_{4}+b_{3}^{2}\right)\left(b_{4} b_{6}+b_{5}^{2}\right) \\
& =b_{2} b_{6} b_{4}^{2}+\left(b_{2} b_{5}^{2}+b_{3}^{2} b_{6}\right) b_{4}+b_{3}^{2} b_{5}^{2} .
\end{aligned}
$$

On the other hand,

$$
\begin{aligned}
b_{1} b_{3} \cdot b_{5} b_{7} & =\left(b_{0} b_{4}-b_{2}^{2}\right)\left(b_{4} b_{8}-b_{6}^{2}\right) \\
& =b_{0} b_{8} b_{4}^{2}-\left(b_{0} b_{6}^{2}+b_{2}^{2} b_{8}\right) b_{4}+b_{2}^{2} b_{6}^{2} .
\end{aligned}
$$

Equating these mod $b_{4}^{2}$ and noticing that $b_{3} b_{5} \equiv b_{2} b_{6}$, we see that everything drops out except the multiples of $b_{4}$. It follows that

$$
-\left(b_{0} b_{6}^{2}+b_{2}^{2} b_{8}\right) \equiv b_{2} b_{5}^{2}+b_{3}^{2} b_{6} \quad\left(\bmod b_{4}\right) \text {. }
$$

But

$$
\begin{aligned}
b_{1} b_{2}\left(b_{2} b_{5}^{2}+b_{3}^{2} b_{6}\right) & \equiv b_{2}^{2} b_{5} \cdot b_{1} b_{5}+b_{1} b_{3}^{2} \cdot b_{2} b_{6} \\
& \equiv b_{2}^{2} b_{5} \cdot b_{3}^{2}+b_{1} b_{3}^{2} \cdot b_{3} b_{5} \\
& \equiv b_{3}^{2} b_{5}\left(b_{2}^{2}+b_{1} b_{3}\right) \equiv 0 \quad\left(\bmod b_{4}\right) .
\end{aligned}
$$

Thus $b_{2} b_{5}^{2}+b_{3}^{2} b_{6} \equiv 0\left(\bmod b_{4}\right)$, and hence

$$
b_{0} b_{6}^{2}+b_{2}^{2} b_{8} \equiv 0 \quad\left(\bmod b_{4}\right) \text {, }
$$

as we wished to prove. 
For $\operatorname{Somos}(5)$, we shall prove that

$$
a_{n} a_{n+7} a_{n+8}+a_{n+2} a_{n+3} a_{n+10} \equiv 0 \quad\left(\bmod a_{n+5}\right) .
$$

This shows that if $a_{n}$ is prime to $a_{n+5}$ then $a_{n+5}$ is prime to $a_{n+10}$. Since $a_{n}$ is prime to $a_{n+5}$ for $n=0,1,2,3,4$, the general result follows by induction.

Again put $b_{i}=a_{n+i}$. Then we see that

$$
\begin{aligned}
b_{2} b_{7} \cdot b_{3} b_{8} & =\left(b_{3} b_{6}+b_{4} b_{5}\right)\left(b_{4} b_{7}+b_{5} b_{6}\right) \\
& =b_{4} b_{6} b_{5}^{2}+\left(b_{3} b_{6}^{2}+b_{4}^{2} b_{7}\right) b_{5}+b_{3} b_{4} b_{6} b_{7}, \\
b_{1} b_{6} \cdot b_{4} b_{9} & =\left(b_{2} b_{5}+b_{3} b_{4}\right)\left(b_{5} b_{8}+b_{6} b_{7}\right), \\
& =b_{2} b_{8} b_{5}^{2}+\left(b_{2} b_{6} b_{7}+b_{3} b_{4} b_{8}\right) b_{5}+b_{3} b_{4} b_{6} b_{7}, \\
b_{1} b_{4} \cdot b_{6} b_{9} & =\left(b_{0} b_{5}-b_{2} b_{3}\right)\left(b_{5} b_{10}-b_{7} b_{8}\right) \\
& =b_{0} b_{10} b_{5}^{2}-\left(b_{0} b_{7} b_{8}+b_{2} b_{3} b_{10}\right) b_{5}+b_{2} b_{3} b_{7} b_{8} .
\end{aligned}
$$

Forming the alternating sum of these three equations mod $b_{5}^{2}$, we see that all the terms go out except the multiples of $b_{5}$. It follows that

$$
\left(b_{3} b_{6}^{2}+b_{4}^{2} b_{7}\right)-\left(b_{2} b_{6} b_{7}+b_{3} b_{4} b_{8}\right) \equiv b_{0} b_{7} b_{8}+b_{2} b_{3} b_{10} \quad\left(\bmod b_{5}\right) .
$$

But $b_{3} b_{6} \equiv b_{2} b_{7}$ and $b_{4} b_{7} \equiv b_{3} b_{8}$, so that the left side vanishes mod $b_{5}$. Hence

$$
b_{0} b_{7} b_{8}+b_{2} b_{3} b_{10} \equiv 0 \quad\left(\bmod b_{5}\right) \text {, }
$$

as we wished to prove.

\section{SOME PROPERTIES OF THE PERIODS}

This section presents some results about $\operatorname{Somos}(4)$ and $\operatorname{Somos}(5)$ that were obtained by calculation, but for which I do not have general proofs.

There is no obvious rule for predicting the period mod $p$, but knowing this, the period $\bmod p^{l}$ seems to follow. Indeed, if $p>2$, then the period $\bmod p^{l}$ is just $p^{l-1}$ times the period $\bmod p$. The case $p=2$ is peculiar. The following periods were observed. For $\operatorname{Somos}(4)$, the period is $5 \bmod 2,10 \bmod 4$, and $5 \cdot 2^{l-2} \bmod 2^{l}$ for $l \geqq 3$. For $\operatorname{Somos}(5)$, the period is $6 \bmod 2,12 \bmod 4$ and $\bmod 8$, and $3 \cdot 2^{l-2} \bmod 2^{l}$ for $l \geqq 4$.

For $k=4$ and $k=5$, if a prime $p$ divides any term of $\operatorname{Somos}(k)$, then the multiples of $p$ are equally spaced. (This is not true for $k=6$ or $k=7$.) However, there are many primes that do not occur at all.

Call the interval between two consecutive multiples of $p$ the gap, denoted by $g$. Since one gap is centered at $(k-1) / 2$, it follows that if we find the smallest positive $n$ for which $a_{n}$ is divisible by $p$, then $g=2 n-(k-1)$. Thus the gap is odd when $k=4$ and even when $k=5$. Conversely, if the gap is given then $p$ divides $a_{n}$ just when $n \equiv(g+k-1) / 2(\bmod g)$.

It turns out that the gap is never much larger than $p$. Specifically, for every prime $p<2000$ that occurs as a factor of terms in $\operatorname{Somos}(4)$ or $\operatorname{Somos}(5)$, we have $g<1.1 p+6$. Roughly speaking, some multiple of $g$ is near $p$, but it is hard to make this more precise.

If $p$ occurs as a factor in $\operatorname{Somos}(4)$ or $\operatorname{Somos}(5)$, then the period $\bmod p$ must be a multiple of $g$. It also appears that the period is always a divisor of $(p-1) g$. 
TABLE 1

\begin{tabular}{|r|r|r|r|r|}
\hline & & Somos(4) & Somos(5) & \\
\hline$p$ & Gap & Period & Gap & Period \\
\hline 2 & 5 & 5 & 6 & 6 \\
3 & 7 & 14 & 8 & 16 \\
5 & & 16 & 10 & 40 \\
7 & 9 & 54 & & 20 \\
11 & 17 & 170 & 12 & 120 \\
13 & & 96 & & 40 \\
17 & & 144 & & 288 \\
19 & & 90 & & 48 \\
23 & 11 & 242 & & 198 \\
29 & & 42 & 34 & 952 \\
31 & & 135 & & 228 \\
37 & & 171 & 14 & 252 \\
41 & 51 & 102 & & 1040 \\
43 & & 147 & 24 & 1008 \\
47 & 57 & 2622 & & 1012 \\
53 & 53 & 1378 & & 1456 \\
59 & 13 & 754 & 24 & 1392 \\
61 & 35 & 350 & 22 & 132 \\
67 & & 495 & & 440 \\
71 & 63 & 4410 & & 2730 \\
73 & 75 & 1350 & 36 & 1296 \\
79 & & 1482 & & 5460 \\
83 & 33 & 2706 & 16 & 1312 \\
89 & & 3784 & 46 & 4048 \\
97 & 47 & 376 & & 138 \\
\hline
\end{tabular}

Table 1 shows, for $\operatorname{Somos}(4)$ and $\operatorname{Somos}(5)$, the gap and period mod $p$ for each prime $p<100$, the gap column being left blank if the prime $p$ does not occur as a factor.

No term of $\operatorname{Somos}(4)$ or $\operatorname{Somos}(5)$ is divisibly by 4 . But for $p>2$, it appears that if the factor $p$ occurs then $p^{2}$ will also occur. In general, the gap $\bmod p^{2}$ seems to be $p$ times the gap $\bmod p$.

However, there is an exceptional case when $k=5$ and $p=73$. Here all terms divisible by $p$ actually have the factor $p^{2}$, so that the gap mod $p^{2}$ is the same as the gap $\bmod p$. If $p^{i}$ is the smallest power of $p$ to occur as a factor, I think that the gap mod $p^{i+l}$ will be $p^{l}$ times the gap mod $p^{i}$.

\section{Periodicity of Generalized Somos Sequences}

There are a number of generalized Somos sequences that admit an alternative recursion formula not involving division. This furnishes not only a proof that the terms of the sequence are integers, but also that the sequences are periodic mod $m$ for every $m$. I do not know such an alternative recursion formula for any $\operatorname{Somos}(k)$. 
We shall consider three sequences, depending on $k$, in this section. The first two sequences were suggested by Dana Scott, the third by David Gale. In each case, we start with the initial values $a_{0}=a_{1}=\cdots=a_{k-1}=1$.

The first sequence, for any $k \geqq 3$, is defined by

$$
a_{n} a_{n-k}=a_{n-1}^{2}+a_{n-2}^{2}+\cdots+a_{n-k+1}^{2} \text { for } n \geqq k .
$$

Here we find that

$$
\frac{a_{n}+a_{n-k}}{a_{n-1} a_{n-2} \cdots a_{n-k+1}}
$$

is an invariant. The equality of this expression for two consecutive values of $n$ is an immediate consequence of the recursion formula. For $n=k$, the invariant has the value $k$. This leads to the alternative recursion formula

$$
a_{n}=k a_{n-1} a_{n-2} \cdots a_{n-k+1}-a_{n-k} \text { for } n \geqq k .
$$

The second sequence, for odd $k \geqq 5$, is defined by

$$
a_{n} a_{n-k}=a_{n-1} a_{n-2}+a_{n-3} a_{n-4}+\cdots+a_{n-k+2} a_{n-k+1} \quad \text { for } n \geqq k .
$$

Here we find that

$$
\frac{a_{n}+a_{n-k-1}}{a_{n-2} a_{n-3} \cdots a_{n-k+1}}
$$

is an invariant. For $n=k+1$, this has the value $k-1$. This leads to the recursion formula

$$
a_{n}=(k-1) a_{n-2} a_{n-3} \cdots a_{n-k+1}-a_{n-k-1} \text { for } n \geqq k+1 .
$$

Notice that this involves an earlier term than the original recursion formula.

The third sequence, for $k \geqq 4$, is defined by

$$
a_{n} a_{n-k}=a_{n-1} a_{n-2}+a_{n-2} a_{n-3}+\cdots+a_{n-k+2} a_{n-k+1} \quad \text { for } n \geqq k .
$$

Here we find that

$$
\frac{a_{n}+a_{n-2}+a_{n-k+1}+a_{n-k-1}}{a_{n-2} a_{n-3} \ldots a_{n-k+1}}
$$

is an invariant. For $n=k+1$, this has the value $2 k-2$. This leads to the recursion formula

$$
a_{n}=(2 k-2) a_{n-2} a_{n-3} \cdots a_{n-k+1}-a_{n-2}-a_{n-k+1}-a_{n-k-1} \text { for } n \geqq k+1 \text {. }
$$

Although the invariant in the third case is the most complicated, it was the first to be found. I discovered the invariant for this sequence with $k=4$ when I was looking for a proof that the sequence has integer terms. Dean Hickerson pointed out that the invariant could be extended to arbitrary $k$. After that, I found the invariants for the other sequences considered.

\section{RATE OF GROWTH OF SOMOS SEQUENCES}

This section is unrelated to the main theme of this paper, which is the periodicity of various sequences mod $m$. However, the growth patterns of Somos sequences are so extraordinary that they deserve mention. The conclusions here are based on numerical calculations, but proofs are lacking.

Consider some $\operatorname{Somos}(k)$, or any variant obtained by altering the initial values to arbitrary positive integers. It appears that there exist positive constants 
$A, B, C$, and a real constant $D$ such that, for all positive and negative values of $n$, we will have

$$
A C^{(n-D)^{2}}<a_{n}<B C^{(n-D)^{2}} .
$$

If the initial values are all 1 's, or more generally any sequence that reads the same backwards as forwards, then the sequence $a_{n}$ has a center of symmetry at $(k-1) / 2$, so that we must have $D=(k-1) / 2$. In other cases, the value of $D$ is not easy to predict. For example, if we modify $\operatorname{Somos}(4)$ by putting $a_{3}=3$, then the value of $D$ is approximately -0.003491 .

Furthermore, $a_{n} / C^{(n-D)^{2}}$ has a periodic oscillation. For $\operatorname{Somos}(4)$, the period is about 2.6391. Indeed, I found that

$$
a_{n} /(1.1076302425)^{(n-3 / 2)^{2}}-0.92293
$$

has the same sign as

$$
\cos (2 \pi(n-3 / 2) / 2.6391)
$$

for all $n$ with $|n|<6000$ and remains rather close to 0.141 times this cosine.

Although the constants so chosen may not be the correct asymptotic values, it still seems remarkable that such a choice is possible. However, it appears that even with the best choice of the constants, some further correction of a smaller order will be necessary. I do not know what form this correction will take.

\section{ADDED IN PROOF}

Here are some more recent results which I have heard about. Michael Somos has found a proof of periodicity $\bmod m$ for $\operatorname{Somos}(4)$ and $\operatorname{Somos}(5)$ which is quite different from mine (see $\S 2$ ). Clifford S. Gardner has shown that the multiples of a given number in $\operatorname{Somos}(4)$ are equally spaced (see $\S 4$ ), and has found an exact expression in terms of theta functions for the oscillations in $\operatorname{Somos}(4)$ and $\operatorname{Somos}(5)($ see $\S 6)$.

\section{REFERENCES}

1. David Gale, The strange and surprising saga of the Somos sequences, Math. Intelligencer 13 (1991), no. 1, 40-42.

2. Michael Somos, Problem 1470, Crux Mathematicorum 15 (1989), 208.

Department of Mathematics, University of California, Berkeley, California 94720 